\title{
Mapping two measures to the International Classification Of Functioning, Disability and Health and the brief ICF core set for spinal cord injury in the post-acute context
}

\author{
Conran Joseph, Julie Phillips, Kerstin Wahman and Lena Nilsson Wikmar
}

\begin{abstract}
Objective: To evaluate the extent to which the rehabilitation outcome levels (ROL) and the spinal cord independence measure (SCIM) III could be mapped to the International Classification of Functioning, Disability and Health (ICF) and the brief core set for spinal cord injury (SCI) in the postacute context. Methods: Two professionals used the published protocol to map the concepts derived from both measures to the ICF categories. Further, the endorsed categories at the second level of the ICF were used to determine the coverage of the Brief ICF Core Set for SCI. Results: Three items of the ROL could not be conceptualised within the ICF, while the rest were mapped to 42 second-level categories, mainly to the activity and participation domain. All the items of the SCIM III were mapped, yielding 52 ICF categories, mostly at the third level (32). For the mapping to the Core Set for SCI, the ROL covered five and the SCIM III all nine categories of 'activities and participation' included as the candidate categories of the brief version. Conclusion: In terms of content, the ROL appears to be a more global measure of functioning, compared with the SCIM III that covers specific 'activity' aspects as proposed in the Brief Core Set for SCI. It is thus recommended that standardised measures, such as the SCIM III, be used due to its conceptual underpinnings and coverage of important aspects.
\end{abstract}

\section{$\rightarrow$ Implications for rehabilitation}

- Rehabilitation professionals should select appropriately validated outcome measures specific to the health condition in order to evaluate the effectiveness of rehabilitation.

- Rehabilitation professional working with outcome measures should be aware of the limitations of measures, in terms of content, and supplement the evaluation with appropriate standardised measures or the use of the Core Sets.

- To enhance evidence-based practise in routine clinical practise, standardised outcome measures should be used.

\section{Background}

The goal of research in the field of outcome measurement is to identify patient characteristics altered following the onset of a health condition and to determine the efficacy of rehabilitation.[1] For the aforementioned purposes, many outcome measures, 
both disease-specific [2-4] and generic, have been develop and proposed.[5] Over the last two decades, the development of outcome measures for the spinal cord-injured (SCI) population has evolved; however, limited knowledge is available on whether or not the content of certain outcome measures used in clinical settings is based on a conceptual or theoretical framework that usually serves as validation for the inquiry of interest.[6]

In South Africa, the SCI-rehabilitation discipline is under development and the use of outcome measures in care has only gained momentum recently. As such, the implementation of outcome measures in SCI is still decentralised.[7] One instrument in use in SCI - rehabilitation in South Africa is the rehabilitation outcome levels (ROL). The ROL is a generic measure of functioning and is used to determine health and rehabilitation needs as well as to predict prognosis during in-patient rehabilitation. A recent local study that used the ROL found that $68 \%$ of participants achieved community reintegration at the end of in-patient rehabilitation, indicating an optimal level of functioning.[8] However, the aforementioned study did not report on the psychometric properties of the outcome measure. Furthermore, to our knowledge, no other studies are available that 'proofs' the rigour of the measure.

The Spinal Cord Independence Measure (SCIM) version III is an outcome measure that covers similar but not identical constructs as the ROL. The SCIM III has been proposed by many as the functional rating scale of choice due to its sound psychometric properties.[2,911] Similar to the ROL, the first version of the SCIM was developed prior to the universal use of the International Classification of Functioning, Disability and Health (ICF), thus leaving a void in the literature regarding its conceptual underpinnings and targeted functioning aspects.

Since the year 2001, the ICF became the conceptual model for the description of health status and disability.[12] Prior to this, limited consensus persisted in the arena of an operative model to be used for the description of disability and its impact on the individual.[12] Many outcome measures have been developed without the use of the ICF as the conceptual basis and therefore the uncertainty remains whether or not those measures are fit for current practise. This scenario of adopting measures without validating its content could give rise to a possible mismatch between 'what we intend to measure and what we actually measure'.[13] To assist us with what is relevant to evaluate for specific health conditions, certain ICF Core Sets have been developed.[14,15]

Specific to the SCI population, both Comprehensive and Brief ICF Core Sets for the early post-acute and long-term context have been developed,[16,17] which provide us with a framework concerning 'the what' to measure for the relevant assessment of functioning. In this study, the Brief Core Set was chosen for a number of reasons. First, the introduction of outcome measures into the clinical environment should allow healthcare providers to make brief assessment of their patients' functioning in the presence of high workload. Second, the candidate categories covered in the 'activity and participation' domain of the 
Brief ICF Core Set are appropriate to the immediate post-acute/in-patient rehabilitation goals that focus on mobility and self-care. Lastly, the candidate categories of the Brief Core Set were determined based on methodological considerations that controlled for all other categories contained in the Comprehensive ICF Core Set.[18]

With the adoption of outcome measures into the era of the ICF and the existence of ICF core sets for SCI, little is known about the conceptual basis and coverage of important functioning aspects, as proposed by the ICF and the Brief ICF Core Set for SCI in the postacute context, of two outcome measures, of which one is used as part of the clinical routine (ROL) and the other a gold standard measure (SCIM III) not currently utilised in South African SCI-care. The objectives of this study were as follows: (i) to determine extent to which the ROL and SCIM III could be mapped to precise categories of the ICF and (ii) to examine the extent that the endorsed codes from each respective measure covered the candidate categories contained in the Brief ICF Core Set for SCI in the post-acute context.

\section{Methods}

\section{Research design}

The qualitative mapping was applied to study the comparative content validity of two measures in relation to the Brief ICF Core Set for SCI. The published ICF linking of outcome measures protocol and the updated version $[19,20]$ were used to standardise the procedure of qualitatively linking items of both outcome measures to the categories of the ICF and thereafter to the Brief Core Set for SCI in the post-acute context.[16]

\section{The ROL and SCIM III}

The ROL was first published in 1995, prior to the implementation of the ICF. The developers of the ROL argue that the importance of applying outcome levels in the planning process of individual patients lies in the ability of outcome levels to guide goal setting, since lower levels must be reached before higher ones.[21] The ROL consists of six distinct levels, representing different functioning aspects that range from impairment to participation outcomes. Each level is written as a set of criteria that needs to be fulfilled, rather than specific items. These levels are defined as groupings or categories of patient problems and conditions, which according to Landrum et al.,[21] are understood to represent levels of progress along a continuum in the process of rehabilitation. On admission to and discharge from rehabilitation, healthcare professionals are responsible for allocating and recording the most appropriate functioning level of each patient.

The SCIM III is currently considered the gold standard outcome measure of investigating functional abilities in the SCI population due to its favourable psychometric properties. This measure includes 19 items across four functional areas namely: (i) self care, (ii) respiration, (iii) sphincter management and (iv) mobility. Similar to the ROL, the first version of this measure was published prior to the acceptance of the ICF in 2001, and both measures contain concepts related to the activity and participation domain.[2] 


\section{Brief ICF core set for SCI in the post-acute context}

To facilitate the application of the ICF, experts have developed both comprehensive and Brief Core Sets for SCI in the post-acute and long-term context.[17] These Core Sets define the relevant and essential categories of functioning to be evaluated in survivors of SCI. A Comprehensive Core Set for SCI in the post-acute context is available; however, we decided to use the Brief Core Set that includes fewer categories that sufficiently capture the typical spectrum of problems experienced in the functioning of survivors with SCI.[16] The categories in the Brief Core Set include three body structure and eight functions, nine activities/participation and five environmental factors.

\section{Practise and standardisation of the linking procedure}

The two experts (health professionals and academics) that were responsible for the mapping are familiar with the nomenclature of the ICF and its application in research and clinical practise. Prior to the mapping exercise, both experts were required to study the four main linking rules [19] and the updated rules.[20] On the day of the mapping, a practise run was held where another functional rating scale (Barthel Index) was mapped and the process was refined based on emerging issues. Experts were advised to identify and map all the meaningful concepts within each item, including the response options, in the outcome measure to the most precise second and third-level category of the ICF, where applicable. If a single item encapsulated different concepts, the meaningful units in each concept were then linked. In addition, if a concept of an item was not explicitly named in a particular category, the lower level of the category (at the second level) was linked. If the information provided by the meaningful concept was not sufficient to endorse a specific code and category, the concept was assigned 'not definable' (nd).[20]

Apart from linking the meaningful concepts to the categories of the ICF, the identified second-level categories of each item in the outcome measure were mapped to the Brief ICF Core Set for SCI in the post-acute context for evaluating the coverage of pertinent categories.[16]

\section{Data analysis}

The result of the mapping was the extent to which items could be mapped to the second- and third-level categories of the ICF. The number of categories addressed per domain of the ICF of each outcome measure was determined, as well as the interobserver agreement for the endorsement of categories at the two distinct levels. Higher levels of absolute agreement were interpreted as the clarity of the concepts from the measures and the ability to locate a specific category of the ICF to correspond with the desired functioning aspect. To account for chance to influence agreement levels, we computed the Prevalence-Adjusted Bias-Adjusted Kappa (PABAK) [22] at the second and third-level of the ICF. The evaluation of conceptual coverage of measures concerning the categories in Brief ICF Core Set for SCI was done using the second-level categories of the ROL and SCIM III, except for one third-level category of the SCIM III. 


\section{Results}

\section{Mapping of the ROL to the categories of the ICF}

As seen in Table 1, 24 meaningful concepts were identified where duplicates were subtracted rather than removed from the list (e.g. self-care, mobility and communication). Of the 24 concepts (Table 2), 11 that were derived from level 2 (representing physiological maintenance) yielded 20 second-level categories. The 2 concepts from level 3 (residential re-integration), 7 from level 4 (community re-integration) and 4 from level 5 (return to productive activity) were linked to 6,10 and 6 unique second-level categories, respectively. Furthermore, 3 of the 24 concepts were meaningful, namely 'physiological stability', 'self-management' and 'self-directed health monitoring', but could not be mapped to a specific category of the ICF and was thus coded 'nd'. In total, the 21 concepts were mapped to 42 second-level and 3 third-level categories of the ICF. Of the second-level categories, 13 'body structure and function' and 29 'activity and participation' categories were identified. The inter-observer (absolute) agreement (and PABAK) of linking concepts to the second- and third-level categories was 57\% (0.357) and o\% (o.500), respectively.

\section{Mapping of the SCIM III to the categories of the ICF}

In Table 3, the 19 items of the SCIM III were mapped to categories of the ICF. From the items, 20 different (duplicates were removed) second-level categories and 32 (duplicates were removed) third-level were identified. Of the 20 second-level categories, 16 were classified in the 'activity and participation' domain. Of the 32 third level categories, 25 were derived from the 'activity and participation' domain. The inter-observer (absolute) agreement (and PABAK) of linking concepts to the second and third-level categories was $80 \%(0.700)$ and $72 \%(0.591)$, respectively. 


\begin{abstract}
Rehabilitation outcome level
Level 0: Physiological instability ${ }^{a}$. Usually occurs early after onset in an acute setting. Assessment, diagnosis and management of medical conditions are ongoing. Includes unmanaged medical problems presenting at a later stage, e.g. pressure sores/inadequate bladder management.

Level 1: Physiological stability ${ }^{a}$. Medical problems addressed and appropriately managed. Condition is stable - no longer requires acute care setting.

Level 2: Physiological maintenance ${ }^{\mathrm{b}}$. Management plans in place to ensure ongoing maintenance of skin integrity, respiration, nutrition, range of movement and bowel and bladder care. Basic functional goals at this stage should include bed mobility, self-care and communication, cognition and behaviour.
\end{abstract}

Level 3: Residential re-integration ${ }^{\mathrm{b}}$. Safe functioning at home. Includes: Physiological stability, self-care, mobility around home, effective communication, simple housekeeping, household planning and management.

Level 4: Community re-integration ${ }^{\mathrm{b}}$. Subject functions appropriately in the community. Includes: self-management, self-directed care of health, ability to function socially, community mobility, recreational activities, community activities, complex home management, financial management and safety in the community.
Level 5: Return to productive activity ${ }^{\mathrm{b}}$. It includes a focus on vocational, a vocational or educational pursuits, household management, school or employment. Productive activities within the patients' level of ability. Includes paid work, unpaid work, volunteer work and education/training.
Concepts identified

No specific meaningful concepts related to the categories of the ICF were identified.

- Unmanaged medical problems

- Identifying immediate and secondary complications

No specific meaningful concepts related to the categories of the ICF were identified

- Medical problems identified and managed

Patient presents with safe systems of:

- Nutrition

- Respiration

- Skin preservation

- Joint maintenance

- Bladder and

- Bowel management

Functional goals include:

- Self-care

- Bed mobility

- Communication

- Cognition

- Behaviour

Goals include proficiency in:

- Self-care

- Mobility in and around dwelling

- Communication

- Home keeping and management

- Physiological stability ${ }^{c}$

Goals include:

- Self-management ${ }^{c}$

- Social competencies

- Community mobility

- Financial management

- Self-directed health monitoring

- Participation in sport, recreation and community activities Independence in:

- Self-care

- Mobility

- Communication

- Community integration

Patient should be able to:

- Engage in vocational and educational pursuits

- Household management

- Attend school or

- Work (paid/unpaid or volunteering)

aLevels 0 and 1, no meaningful concepts consistent with the categories of the ICF and Brief Core Set for SCl in the post-acute context have been identified. The terms 'unmanaged medical problems', 'identify immediate and secondary complications' and 'medical problems identified and managed' only denote meaningful units qualitatively.

${ }^{b}$ Combining levels 2-5, 24 unique meaningful concepts were identified (excluding duplicate concepts), of which 21 were relevant for the mapping to the ICF categories.

'These items were meaningful but no ICF codes were endorsed.

\section{Mapping of ROL and SCIM III to the brief ICF core set for SCI}

As evident in Table 4, the ROL covered 4 of the 11 impairments (body function and structure) and 5 of the 9 'activity and participation' categories in the Brief ICF Core Set for SCI. Considering the coverage of the SCIM III, 3 of the 11 impairments and all 9 'activity and participation' categories were addressed. Concerning 'activity' items in the ROL, the item 'self-care' seemed to lack a clear definition as the two experts only agreed on its implied meaning to be related to the category 'washing oneself' (d510). Further, no agreement was found when 'self-care' was also mapped to the category 'toileting ( $\mathrm{d}_{530}$ ) and dressing (d540)'. In the ROL, self-care is used as an umbrella term for multiple tasks, and no distinction was made concerning dressing and bathing of the upper and lower body, as evident in the SCIM III. The results further demonstrate that none of the measures contains items related to environmental categories. 


\begin{tabular}{|c|c|c|c|}
\hline Items ROL & Second-level codes & $\begin{array}{l}\text { Third-level } \\
\text { codes }\end{array}$ & Second-level category \\
\hline \multicolumn{4}{|c|}{$\begin{array}{l}\text { Level } 0 \text { and 1: Presence of unmanaged } \\
\text { medical problems Identifying immediate and } \\
\text { secondary complications Medical problems } \\
\text { identified and managed }\end{array}$} \\
\hline Nutrition & $\mathrm{b} 530$, b510, b515 & & $\begin{array}{l}\text { Weight maintenance function; ingestion functions; digestive } \\
\text { functions }\end{array}$ \\
\hline Respiration & b440 & & Respiration functions \\
\hline Skin preservation & b810, b840 & & Protective functions of the skin; sensation related to the skin \\
\hline Joint maintenance & b710, b715 & & Mobility of joint functions; stability of joint functions \\
\hline Bladder and & b610 & & Urinary excretory functions \\
\hline Bowel management & b525 & & Defecation functions \\
\hline Functional goals include; Self- care & $\mathrm{d} 510, d 520, d 530, d 540$ & & Washing oneself; caring for body parts; toileting; dressing \\
\hline Bed Mobility & $\mathrm{d} 410$ & & Changing basic body position \\
\hline Communication & $\mathrm{d} 310, \mathrm{~d} 315$ & & $\begin{array}{l}\text { Communicating with-receiving-spoken messages; non-verbal } \\
\text { messages }\end{array}$ \\
\hline Cognition & b110, b164 & & Consciousness functions; high-level cognitive functions \\
\hline Behavior & b126 & & Temperament and personality functions \\
\hline \multicolumn{4}{|l|}{ Level 3: Goals include proficiency in; } \\
\hline Self-care & $\mathrm{d} 510, d 520, d 530, d 540$ & & Washing oneself; caring for body parts; toileting; dressing \\
\hline Mobility in and around dwelling & d450 & & Walking \\
\hline Communication & $\mathrm{d} 310, \mathrm{~d} 315$ & & $\begin{array}{l}\text { Communicating with-receiving-spoken messages; non-verbal } \\
\text { messages }\end{array}$ \\
\hline Home keeping and management & $\mathrm{d} 620, \mathrm{~d} 630, d 640, d 650, d 660$ & & $\begin{array}{l}\text { Acquisition of goods and services; preparing meals; doing } \\
\text { housework; caring for household objects; assisting others }\end{array}$ \\
\hline Physiological stability & nd & & \\
\hline \multicolumn{4}{|l|}{ Level 4: Goals include; } \\
\hline Self-management & nd & & \\
\hline Social competencies & $\mathrm{d} 710, d 720, d 730, d 740, d 750$ & & $\begin{array}{l}\text { Basic interpersonal interactions; complex interpersonal inter- } \\
\text { actions; relating with strangers; formal relationships; informal } \\
\text { social relationships }\end{array}$ \\
\hline Community mobility & d465 & & Moving around using equipment \\
\hline Financial management & $\mathrm{d} 860, \mathrm{~d} 865$ & $d 8708$ & Basic economic transactions; complex economic transactions \\
\hline Self-directed health monitoring & nd & & \\
\hline $\begin{array}{l}\text { Participation in sport, recreation and } \\
\text { community activities }\end{array}$ & d910, d920 & & Community life; recreation and leisure \\
\hline Independence in; Self-care & $\mathrm{d} 510, d 520, d 530, d 540$ & & Washing oneself; caring for body parts; toileting; dressing \\
\hline Mobility & d450 & & Walking \\
\hline Communication & $\mathrm{d} 310, d 315$ & & $\begin{array}{l}\text { Communicating with-receiving-spoken messages; non-verbal } \\
\text { messages }\end{array}$ \\
\hline Community integration & $d 910$ & & Community life \\
\hline Level 5: Patient should be able to; & & & \\
\hline $\begin{array}{l}\text { Engage in vocational and educational } \\
\text { pursuits }\end{array}$ & $\mathrm{d} 820, \mathrm{~d} 825$ & & School education; vocational training \\
\hline Household management & d699 & & Domestic life \\
\hline Attend school or & d820 & & School education \\
\hline Work & $\mathrm{d} 840, \mathrm{~d} 845, \mathrm{~d} 850$ & $d 8500, d 8502$ & $\begin{array}{l}\text { Apprenticeship; acquiring, keeping and terminating a job; } \\
\text { remunerative employment }\end{array}$ \\
\hline
\end{tabular}

$\mathrm{ROL}=$ Rehabilitation Outcome Level measure

Codes in italics = Indicate disagreement between the two experts that conducted the mapping

nd $=$ not definable

\section{Discussion}

Many outcome measures in the rehabilitation arena have been developed prior to the acceptance of the ICF framework as the standard language for the description of health and disease. In this study, we aimed to evaluate whether the ROL and the SCIM III could be mapped to the ICF and the functioning aspects contained in the Brief ICF Core Set for SCI in the post-acute context.

The main findings of the study suggested that almost all except for 2 of the 21 concepts from the ROL were linked to second-level categories of the ICF. The lack of identifying third-level categories could be explained based on the broad nature of the contained concepts in the items. For example, the concept 'mobility' which was classified under the 
walking category (d450) could have been conceptualised differently by other health professionals, for instance as 'mobility of the upper body/lower body' or 'using a wheelchair/other assistive device'. The same ambiguity is apparent for the item 'self-care', which was uniformly classified under the 'washing oneself' category ( $\mathrm{d}_{510}$ ) by both experts. Furthermore, three of the meaningful concepts in the ROL could not be linked to the ICF and were thus coded 'not definable' (nd).

Concerning the SCIM III, all the concepts derived from the 19 items were mapped to the ICF, with more third-level than second-level categories identified. The higher level coding demonstrates the explicit nature of the items, which reduces the ambiguity of the task or action under study. Further, the agreement between the examiners was higher for the SCIM III than the ROL. The endorsement of codes to higher-level categories seems not only complex in this study but has been reported elsewhere.[23] These findings stress the need of operational definitions of terms in outcome measures and the possible development of training manuals to standardise the rating procedure and the evaluation of patients. 


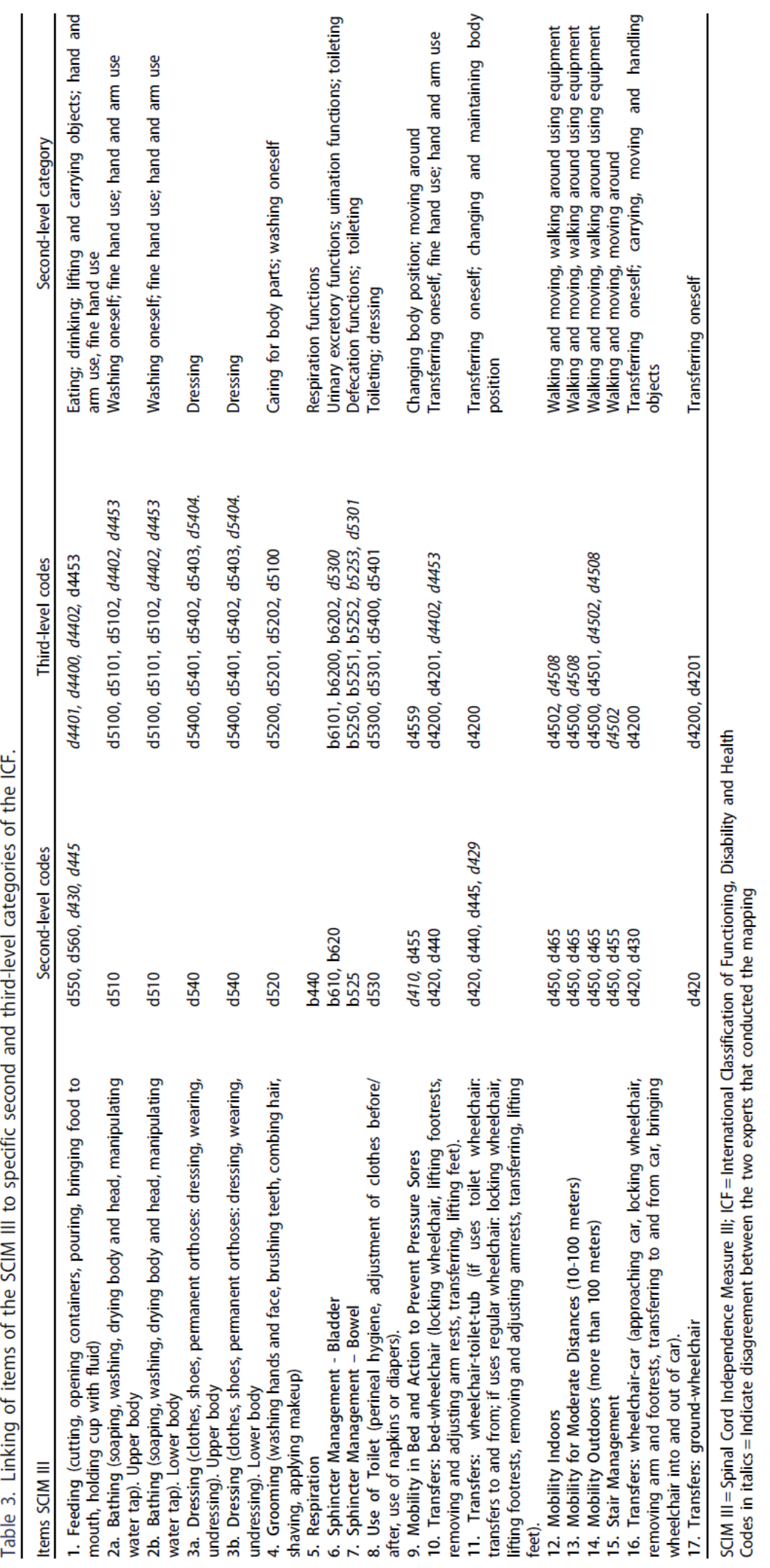




\begin{tabular}{|c|c|c|c|c|}
\hline \multicolumn{3}{|c|}{ Brief ICF core set for $\mathrm{SCl}$} & ROL & \multirow{2}{*}{$\frac{\text { SCIM III }}{\text { Item in measure }}$} \\
\hline Domain & Second-level code & Category & Item in measure & \\
\hline \multirow[t]{8}{*}{ Body functions } & b730 & Muscle power function & & \\
\hline & b620 & Urination function & 'Bladder management' & '6. Sphincter management-bladder' \\
\hline & b525 & Defecation function & 'Bowel management' & '7. Sphincter management-bowel' \\
\hline & b280 & Sensation function & & \\
\hline & b440 & Respiration function & 'Safe systems of respiration' & '5. Respiration' \\
\hline & b735 & Muscle tone function & & \\
\hline & b152 & Emotional function & & \\
\hline & b810 & Protective functions of the skin & $\begin{array}{l}\text { 'Safe system of skin } \\
\text { preservation' }\end{array}$ & \\
\hline \multirow[t]{3}{*}{ Body structures } & s120 & Spinal cord and related structures & & \\
\hline & s430 & Structure of respirator system & & \\
\hline & $s 610$ & Structure of urinary system & & \\
\hline \multirow{13}{*}{$\begin{array}{l}\text { Activities and } \\
\text { Participation }\end{array}$} & $\mathrm{d} 420$ & Transferring oneself & & '10. Transfer from bed to wheelchair' \\
\hline & & & & $\begin{array}{l}\text { '11. Transfer: wheelchair-toilet-tub' } \\
\text { '16. Transfers: wheelchair to car' } \\
\text { '17. Transfers: ground to wheelchair' }\end{array}$ \\
\hline & $\mathrm{d} 410$ & Changing basic body positions & 'Bed mobility' & $\begin{array}{l}\text { '9. Mobility in bed and prevention of } \\
\text { pressure sores' }\end{array}$ \\
\hline & $\mathrm{d} 445$ & Hand and arm use & & $\begin{array}{l}\text { ' } 2 \mathrm{a} \text { and } 2 \mathrm{~b} \text { Bathing (upper and lower } \\
\text { body)': derived from third level } \\
\text { category }\end{array}$ \\
\hline & $\mathrm{d} 530$ & Toileting & 'Self-care' & '8. Use of toilet' \\
\hline & d550 & Eating & & '1. Feeding' \\
\hline & $\mathrm{d} 450$ & Walking & 'Mobility' & '12. Mobility indoors' \\
\hline & & & & $\begin{array}{l}\text { '13. Mobility for moderate distances' } \\
\text { '14. Mobility outdoors' } \\
\text { '15. Stair management' }\end{array}$ \\
\hline & $\mathrm{d} 510^{\mathrm{a}}$ & Washing oneself & 'Self-care' & '2a. Bathing upper body' \\
\hline & & & & '2b. Bathing lower body' \\
\hline & d5 540 & Dressing & 'Self-care' & '3a. Dressing of upper body' \\
\hline & & & & '3b. Dressing of lower body' \\
\hline & d560 & Drinking & & '1. Feeding (also includes drinking)' \\
\hline \multirow[t]{5}{*}{ Environmental factors } & e310 & Immediate family & & \\
\hline & e355 & Health professionals & & \\
\hline & e115 & $\begin{array}{l}\text { Products and technology for personal use in } \\
\text { daily living }\end{array}$ & & \\
\hline & e120 & $\begin{array}{l}\text { Products and technology for personal indoor } \\
\text { and outdoor mobility and transportation }\end{array}$ & & \\
\hline & e340 & $\begin{array}{l}\text { Personal care providers and personal } \\
\text { assistants }\end{array}$ & & \\
\hline
\end{tabular}

ROL, Rehabilitation Outcome Levels measure; SCIM III, Spinal Cord Independence Measure version III; d510 ${ }^{\mathrm{a}}$, Indicates that for mapping of the self-care item (only for ROL), both experts agreed only on this code; shaded grey, indicates that no items in the outcome measure corresponded to the category in the Brief Core Set for $\mathrm{SCl}$ in the post-acute context.

Subsequent to the endorsement of codes of items in the ROL and SCIM III, the secondlevel categories identified from each measure were used to determine the extent of coverage of the Brief ICF Core Set for SCI in the post-acute context. The ROL showed limited coverage of the 'activity and participation' candidate categories contained in the Brief ICF Core Set, addressing five out of the nine. On the contrary, the SCIM III covered all nine 'activity and participation' categories, providing full coverage of the domain in the Brief ICF Core Set for SCI in the post-acute context. In addition to covering more pertinent aspect of functioning, the SCIM III distinguished between upper body and lower body proficiency for items concerning bathing and dressing, aspects not captured in the ROL or any other functional rating scale used in SCI.[24,25] The findings imply that the SCIM III can be used in the clinical context, as earlier suggested,[9-11] but now with proven content validation. Conceptually, the ICF and Core Sets do not only allow us to validate constructs of measurement but also provide the platform to evaluate which functioning domains are represented in measures. Although the purpose of some measures, such as the SCIM III, was not designed to cover the entire spectrum of 
functioning problems but rather one domain/construct, the ICF is capable of identifying the extent to which the relevant categories are covered or not included. Contrary to the SCIM III, the ROL was designed to represent functioning aspects along the continuum of the health condition, i.e. impairment to activity and participation aspects, and claims to be grounded within the bio-psychosocial model.[21] However, we found gaps in coverage of pertinent aspects for persons with SCI in the impairment and activity domain, but most specifically within the environmental factors domain. The ICF is clear when dealing with 'involvements in life situations', emphasising the consideration of the environmental influence on the functioning of individuals.

The results of this study suggest that the ROL is a more global measure of functioning, covering a wider spectrum of ICF categories of activity and participation than the SCIM III. However, more specific to SCI, the SCIM III allows for a more detailed assessment of aspects, e.g. mobility, and is more 'targeted' to categories included in the Brief ICF Core Set than the ROL. As a result, we know little about the extent to which outcomes are addressed and how survivors of SCI describe their perceived involvement in life situations, which hinder the alignment of rehabilitation structures and processes towards optimal patientoriented outcomes. We initiated an ongoing qualitative study that is explorative in nature and aims to provide a broad and thick description of the life areas still affected after rehabilitation and the factors influencing the survivors' self-perceived participation. We will further evaluate the extent to which the identified concepts are covered within the ICF and more specifically the ICF Core Sets for SCI in the appropriate context. These projects could assist in the development of appropriate measures for the context and justify the use of the ICF in healthcare service delivery.

The study presents with some limitations. More independent health professionals at the centre could have been used for the mapping of the ROL since limited information is available concerning the possible hidden meaning of concepts in the measure. This could have enhanced the internal validity of the endorsed codes and categories relevant for each measure. In addition, the Comprehensive ICF Core Set for SCI in the post-acute context could have been used to provide a wider range of categories for the items to be mapped to. However, that would have been merely a theoretical exercise. We believe that the Brief Core Sets are administratively more practical in the 'real-world' setting compared with the Comprehensive ICF Core Sets.

In conclusion, the ROL appears to be a more global measure of functioning that covers a wider spectrum of activity and participation categories of the ICF (although with less preciseness) than the SCIM III. With regard to SCI assessment of functioning, the SCIM III covered all the candidate categories of activity and participation in the Brief ICF Core Sets for SCI, indicating its suitability for this population, while the ROL presented with limitations. Moreover, both measures do not cover environmental aspects; therefore, the assessment of functioning should be supplemented with a measure capturing this contextual component. 


\section{Acknowledgements}

We would like to thank Professor Karin Harms-Ringdahl for her valuable input throughout the writing of this article.

\section{Declaration of interest}

The authors declare that they have no competing interests. 


\section{References}

1. Patrick DL, Chiang YP. Measurement of health outcomes in treatment effectiveness evaluations: conceptual and methodological challenges. Med Care. 2000;38:14-25.

2. Catz A, Itzkovich M, Agranov E, et al. SCIM-spinal cord independence measure: a new disability scale for patients with spinal cord lesions. Spinal Cord. 1997;35:850856.

3. van Hedel H, Wirz M, Dietz V. Assessing walking ability in subjects with spinal cord injury: validity and reliability of 3 walking tests. Arch Phys Med Rehabil. 2005;86:190-196.

4. Field-Fote EC, Fluet GG, Schaefer EM, et al. The spinal cord injury functional ambulation inventory (SCI-FAI). J Rehabil Med. 2001;33:177-181.

5. Buck D, Jacoby A, Massey A, et al. Evaluation of measures used to assess quality of life after stroke. Stroke. 2000;31:2004-2010.

6. Ullrich PM, Spungen AM, Atkinson D, et al. Activity and participation after spinal cord injury: state-of-the-art report. J Rehabil Res Dev. 2012;49:155-174.

7. Inglis G, Faure MR, Frieg A. The awareness and use of outcome measures by South African physiotherapists. South Afr Soc Physiother. 2008;64:5-11.

8. Hassan SAM, Visagie S, Mji G. The achievement of community integration and productive activity outcomes by CVA survivors in the Western Cape Metro Health District. South Afr J Occup Ther. 2012;42:11-16.

9. Anderson KD, Acuff ME, Arp BG, et al. United States (US) multi-center study to assess the validity and reliability of the Spinal Cord Independence Measure (SCIM III). Spinal Cord. 2011;49:880-885.

10. Bluvshtein V, Front L, Itzkovich M, et al. SCIM III is reliable and valid in a separate analysis for traumatic spinal cord lesions. Spinal Cord. 2011;49:292-296.

11. Itzkovich M, Gelernter I. Biering-Sorensen, et al. The Spinal Cord Independence Measure (SCIM) version III: reliability and validity in a multi-center international study. Disabil Rehabil. 2007;30:1926-1933.

12. WHO. ICF, towards a common language for functioning, disability and health. Geneva: WHO; 2002.

13. Wade DT. Measurement in neurological rehabilitation. New York: Oxford University Press; 1992.

14. Cieza A, Ewert T, Ustun B, et al. Development of ICF Core Sets for patients with chronic conditions. J Rehabil Med. 2004;44:9-11.

15. Ewert T, Grill E, Bartholomeyczik S, et al. ICF Core Set for patients with neurological conditions in the acute hospital. Disabil Rehabil. 2005;27:367-373.

16. Kirchberger I, Cieza A, Biering-Sorensen F, et al. ICF Core Sets for individuals with spinal cord injury in the early post-acute context. Spinal Cord. 2010;48:297-304.

17. Cieza A, Kirchberger I, Biering-Sørensen F, et al. ICF Core Sets for individuals with spinal cord injury in the long-term context. Spinal Cord. 2010;48:305-312.

18. Grill E, Stucki G. Criteria for validating comprehensive ICF Core Sets and developing Brief ICF Core Set versions. J Rehabil Med. 2011;43:87-91. 
19. Cieza A, Brockow $T$, Ewert $T$, et al. Linking health-status measurements to the international classification of functioning, disability and health. $J$ Rehabil Med. 2002;34:205-210.

20. Cieza A, Geyh S, Chatterji S, et al. ICF linking rules: an update based on lessons learned. J Rehabil Med. 2005;37:212-218.

21. Landrum P, Schmidt N, Mclean A. Outcome oriented rehabilitation. Gaithersburg, MD: Aspen; 1995.

22. Sim J, Wright CC. The Kappa statistics in reliability studies: use, interpretation and sample size requirements. Phys Ther. 2005;85:257-268.

23. Morriello C, Byrne K, Cieza A, et al. Mapping the Stroke Impact Scale (SIS-16) to the International Classification of Functioning, Disability and Health. J Rehabil Med. 2008;40:102-106.

24. Granger CV, Hamilton BB, Zielezny M, et al. Advances in functional assessment in medical rehabilitation. Top Geriatr Rehabil. 1986;1:59-74.

25. Anderson K, Aito S, Atkins M, et al. Functional recovery measures for spinal cord injury: an evidence-based review for clinical practice and research. J Spinal Cord Med. 2008;31:133-144. 